\title{
Lo biopolítico desde la noción simondoniana de "vida"
}

\author{
The biopolitics from simondonian notion of "life"
}

FRANCISCO VÁZQUEZ MANZANO*

\begin{abstract}
Resumen: A partir de las reflexiones de Michel Foucault en torno al poder, la noción de biopolítica ha sido un topos común en el debate filosófico contemporáneo. Sin embargo, como ya advirtiese Giorgio Agamben, no es posible una comprensión de lo biopolítico sin un enriquecimiento de la noción de "vida" manejada por Foucault. A esta tarea se encomienda principalmente este trabajo, a través de un análisis de la noción de vida propuesta por Gilbert Simondon que permita arrojar luz sobre la naturaleza de lo biopolítico.

Palabras clave: Biopolítica; Vida; Poder; Foucault: Simondon.
\end{abstract}

\begin{abstract}
From the reflections about the power made by Michel Foucault, the notion of biopolitic has been a topos in the contemporary philosophic discussion. However, as Giorgio Agamben did warn, it is not possible to understand biopolitics without an enrichment of the notion of "life" that shows Foucault. Thus, the main aim of this paper is to carry out an analysis of the notion of life conceived by Gilbert Simondon which allows to throw light on the nature of biopolitics.
\end{abstract}

Key words: Biopolitics- Life- Power- FoucaultSimondon.

\section{Introducción}

En "La inmanencia absoluta" (cfr. Agambem, 2007, 481-522), Giorgio Agamben analiza la nociones de "vida" desarrolladas tanto por Foucault como por Deleuze. Si bien, para Agamben, tal noción es fundamental para comprender la filosofía que se desarrolla a partir de ambos autores, no será sino a partir de la obra de Deleuze desde donde la reformulación de esta noción adquirirá toda la importancia que Agamben le concede como punto de apoyo para una filosofía del presente y, sobre todo, para un análisis de los procesos de la subjetivación.

Tras una breve descripción de la noción de vida de Foucault, Agamben emprende un análisis de la propuesta de Deleuze. Lo que Deleuze habría notado es la necesidad de incorporar la vida como "tercer eje" -junto al saber y al poder- desde el cual comprender la realidad del sujeto. Si Foucault lleva a cabo una explicación de la constitución del sujeto en base a los ejes saber-poder, Deleuze, a través de un enriquecimiento de la noción de "vida" que Foucault maneja, muestra la necesidad de incorporar a ésta como "tercer eje" para el cometido que Foucault había perseguido.

Recibido: 09/10/2016. Aceptado: 18/05/2017.

* Universidad de Sevilla, España. Personal de gestión del grupo "Equipo de filosofía de la cultura" (HUM-153), de la facultad de Filosofía. Principales líneas de investigación: pensamiento de la diferencia, hermenéutica y teoría crítica. Correo electrónico: fco_vazque_93@hotmail.com 
Además de ofrecer una correcta explicitación de la noción deleuziana de vida, Agamben trata de enlazar esta cuestión y la del saber, contribuyendo al proyecto de una comprensión del sujeto desde los tres ejes saber-poder-vida. En este artículo se persigue contribuir a ese proyecto, conectando el eje de la vida con ese otro eje que en el trabajo de Agamben queda en segundo plano: el poder. Sin embargo, para este cometido, preferimos realizar un enfoque más radical y, en lugar de centrarnos en la noción deleuziana de vida, partimos de la explicación del proceso de individuación de lo real que Simondon realiza, de la que se desprende el concepto de vida que maneja Deleuze. Este cambio en las fuentes se debe a que, desde dicha explicación, es posible una mejor comprensión del poder tal y como Foucault lo concibe, esto es, como presentando una naturaleza biopolítica.

En la obra de Foucault lo biopolítico se entiende como el hacerse cargo el gobierno de la actividad vital de los individuos. En este sentido, la biopolítica se cifra en una práctica gubernamental caracterizada por ejercer un "poder sobre la vida" que se ejerce a través de lo que Foucault denomina "dispositivos". Para Foucault, tal y como observa Agamben, un dispositivo es "un conjunto heterogéneo que compone los discursos, las instituciones, las decisiones reglamentarias, las leyes..." (cfr. Ibíd., 250). A su vez, el dispositivo tiene una función estratégica dominante, lo que se traduce en cierta manipulación de las relaciones de fuerza, ya sea para desarrollarlas en tal o cual dirección, ya sea para bloquearlas, estabilizarlas o utilizarlas. El dispositivo también está ligado a un límite o a los límites del saber, que le dan nacimiento pero, ante todo, lo condicionan.

Así, en resumen, el dispositivo consiste en una modulación estratégica de las relaciones de fuerza, sostenida por tipos de saber y que modula la propia vida de los individuos. Los dispositivos, así entendidos, se encontrarían asociados a procesos de subjetivación por los cuales se estructura un plano de fuerza que es representado por la "nuda vida". Así pues, son estos dispositivos los encargados de efectuar la estructuración o control sobre la vida que caracterizan a la práctica biopolítica según Foucault.

Desde esta breve consideración, la estructuración biopolítica se presenta como un control -ejercido por el poder- sobre la vida de los individuos que componen una sociedad determinada para amoldar tal "vida desnuda" a una cualificación de la misma. Sin embargo, siguiendo la recomendación de Agamben, es necesario un enriquecimiento de esta noción de vida que permita una mejor comprensión de este tipo de poder que toma a aquella como objeto sobre el cual ejercerse y que con el saber, y la propia vida, constituye uno de los tres ejes de los procesos de subjetivación. En concreto, es necesario mostrar como la propia dinámica de la vida es tal que exige lo biopolítico como un complemento necesario para su desarrollo, al menos, en el ser humano.

En este artículo, dicha tarea se realiza mediante el análisis simondoniano del proceso de individuación. Sin embargo, en este análisis, además de alcanzar un concepto inteligible de "vida" y, así, comprender la naturaleza biopolítica del poder, se alcanzarán las siguientes conclusiones: 1) la inevitabilidad de la estructuración biopolítica en la constitución de la subjetividad humana y 2) que tal estructuración presenta el riesgo de convertirse en una forma patológica de poder.

Para Simondon, la vida se presenta como un nivel de individuación que, con la aparición de lo psíquico, adquiere un nivel de problematicidad para cuya resolución necesita una nueva individuación que estructure ese suelo problemático: nos referimos a la individuación 
colectiva. La tesis que se sostendrá es que tal estructuración del suelo problemático vital en base la individuación colectiva presenta los rasgos de lo biopolítico entendido en sentido foucaultiano, por lo que desde aquí podremos entender mejor la relación que se establece entre el poder y la vida en el proceso de subjetivación del individuo humano.

\section{Lo biopolítico como individuación psico-colectiva}

\subsection{La individuación como resolución y mantenimiento de una problemática originaria}

El objetivo fundamental que recorre la obra de Simondon es una explicitación del proceso mediante el cual se genera realidad individuada. Según el francés, para dar cuenta de tal proceso, es necesario partir de la vigencia de la clásica distinción entre natura naturans como plano intensivo y pre-individual de lo real y natura naturata como la manifestación de ese plano intensivo en una realidad extensiva individuada, ya que de no asumir esta distinción se corre el riesgo de partir de una realidad ya individuada como origen del proceso de individuación (cfr. Simondon, 2009, 36).

Así, el proceso de individuación ha de partir de una realidad pre-individual presta a su individuación. Este plano de realidad pre-individuada Simondon lo concibe como un sistema energético en estado de "meta-estabilidad" (cfr. Ibíd., 82). La meta-estabilidad de un sistema se corresponde con un estado de dicho sistema en el cual las diferencias de potencial intensivo que lo componen se encuentran en un equilibrio tensional-problemático, muy diferente al equilibrio que se considera desde la noción tradicional de estabilidad (cfr. Ibíd., 82).

Precisamente, la individuación se concibe como la aparición de una singularidad por y desde ese conjunto diferencial de potenciales en equilibrios meta-estable -lo que no sería posible partiendo de un sistema en equilibrio estable-. Cuando tal conjunto diferencial adquiere una problematicidad tal que excede el equilibro meta-estable provisorio en el que se encontraba es necesaria la aparición de nuevas formas de equilibrio que contengan la problematicidad creciente. En tal estado, el sistema pierde su equilibrio y pasa a constituir el sistema individuante pre-individual para una nueva individuación. Para Simondon, esta nueva forma de equilibrio generada por el sistema individuante implica una ralentización o "neotenización" de su problemática, la cual se engloba bajo una nueva forma de meta-estabilidad.

Ahora bien, en el momento en el que se produce la actualización del sistema por la aparición de una singularidad que engloba el sistema de base en un nuevo equilibrio metaestable, el sistema permanece en dicha actualización como el medio asociado del individuo constituido (cfr. Ibíd., 83). De modo que el individuo, en sentido estricto, se entiende como un par conformado por la estructura que la actualización confiere al sistema individuante y el medio asociado a tal estructura, entendido éste como el mantenimiento del sistema individuante en el seno de la nueva forma de equilibrio meta-estable. Es por ello por lo que Simondon puede decir que "la individuación es un acontecimiento y una operación en el seno de una realidad más rica que el individuo que resulta de ella" (Ibíd., 87).

1 Para una mayor comprensión de la noción de "equilibrio meta-estable", con la que Simondon pretende corregir la visión clásica de los sistemas que sólo concibe el equilibrio como estable o inestable: cfr. Simondon, 2009, 28-34. 
Por otro lado, todo este proceso de individuación presenta una dinámica transductiva. Así define Simondon la "transductividad":

Una operación física, biológica, mental, social, por la cual una actividad se propaga progresivamente en el interior de un dominio, fundando esta propagación sobre una estructuración del dominio operada aquí y allá: cada región de estructura constituida sirve de principio de constitución a la región siguiente, de modo que una modificación se extiende así progresivamente al mismo tiempo que dicha operación estructurante (Ibíd., 38).

Esa "estructuración del dominio" sería vista como la realidad ya individuada. No obstante, tal realidad individuada lastraría consigo la problemática que le dio origen como su medio asociado, siendo ese "medio" el motor para nuevas individuaciones y constituyendo esta individuación ya efectuada, el suelo pre-individual para una individuación futura. En este proceso se cifra la transducción: la amplificación de una problemática inicial a través de su estructuración y mantenimiento progresivo y nunca acabado.

Desde aquí, es posible entender la relación que guardan, para Simondon, los tres órdenes de individuación: el físico, el vital y el colectivo-transindividual: un equilibrio meta-estable estrictamente físico se rompe por un aumento de tensión entre los potenciales energéticos que engloba dando paso a una singularidad que restablece el equilibrio en nuevo nivel de individuación vital. Este nuevo nivel supone una ralentización o neotenización del nivel físico: la problemática física no se anula, forma parte del nuevo nivel vital siguiendo un devenir heterogéneo respecto a éste. Del mismo modo se produce el paso de la individuación vital a la colectiva trans-individual con una serie de particularidades que en el apartado siguiente examinaremos. ${ }^{2}$

Simondon extrae este paradigma de la individuación en general de la individuación de los seres físico. Pese a que pueda aparentarlo, no nos encontramos ante un reduccionismo, tal y como señala Muriel Combes:

Que l'on puisse tirer des sciences physiques un paradigme qui constitue en quelque sorte un schéma directeur pour l'étude de l'individuation, cela ne signifie pas que l'on prétende « opérer une réduction du vital au physique » lorsqu' on le transpose dans le domaine du vivant (...) On passe d'un domaine d'être à un autre par le transfert des opérations d'une structure à une autre, en ajoutant à chaque niveau les spécificités que le paradigme physique, trop simple, ne permet pas de saisir. Pourtant, le paradigme physique demeure, à titre de paradigme élémentaire (Combes, 1999, 18).

La noción de vida aquí manejada nos permite relacionar el pensamiento de Simondon con el de Foucault. En efecto la vida se concibe como la individuación de un suelo problemático representado por el individuo físico que, a su vez, mantiene esa problemática en su seno. En

2 La relación entre la individuación física y vital también tiene particularidades propias. Dado que este artículo pretende establecer meramente la relación que guardan el nivel de individuación psico-colectivo con una estructuración biopolítica tal y como lo concibe Foucault, una exposición pormenorizada del paso de una individuación estrictamente física a una vital sobrepasa el objetivo marcado. Invitamos al lector interesado a examinar lo dicho sobre tal relación por el propio Simondon en: Simondon, 2009, pp. 118-130 y 177-189 
el momento en el que esa problemática pase a primer plano a través de la aparición de lo psíquico -como explicitamos en el apartado siguiente-, el equilibrio meta-estable estrictamente vital generará una problemática tal que no será posible solventar desde la propia dinámica

vital y que exigirá una nueva individuación. Tal estructuración del suelo vital problemático, presumimos, radica en una cualificación de esa "vida desnuda", en una estructuración de la misma, algo que se entiende desde la propia dinámica transductiva de lo real.

Así, el poder biopolítico que describe Foucault es exigido por la propia vida como una estructuración de la misma que permita la amplificación de su problemática intrínseca, continuando así el proceso de individuación, puesto que, con la aparición de lo psíquico, la vida se estanca en una problemática que no puede solventar por sí misma. De este modo, comprenderemos mejor la relación entre poder y vida que se da en el proceso de subjetivación.

Volviendo a Simondon, la individuación psico-colectiva es una etapa del proceso de individuación. Para el francés, la individuación psico-colectiva se produce cuando la unidad vital, plantea una problemática que no puede resolverse desde ella misma sino que exige, por el contrario, la aparición de un nuevo plano (el colectivo) para resolverla. En el subapartado siguiente trataremos de especificar 1) en qué consiste la unidad representada por la vida, 2) cómo esa unidad se torna problemática, 3) la necesidad de la dimensión psicocolectiva para la resolución de esa problemática vital; y 4) cómo ese efecto que la dimensión psico-colectiva ejerce sobre la vida presenta los rasgos de una estructuración biopolítica tal y como Foucault la concibe.

En base a 1) obtendremos la noción de vida desde la cual comprender la naturaleza de lo biopolítico. 2) supone un enriquecimiento del modo en el que Foucault entiende el operar del poder biopolítico, debido a la introducción de lo psíquico como elemento problematizante de la unidad vital. Por último, 3) y 4) radican en una nueva visión de lo biopolítico a la luz de la noción de vida ya alcanzada, visión que presenta lo biopolítico como elemento necesario en la constitución de la propia subjetividad humana y que si bien, con Foucault, implica cierta restricción y control de la vida de los individuos, también la mantiene en su seno.

\subsection{La individuación vital}

Desde su concepción del proceso de individuación, Simondon entiende la vida como:

una serie transductiva de operaciones de individuación, o también como un encadenamiento de resoluciones sucesivas, pudiendo ser retomada cada resolución anterior y reincorporada en las resoluciones posteriores. Así se podría dar cuenta del hecho de que la vida en su conjunto aparece como una construcción progresiva de formas cada vez más elaboradas, es decir capaces de contener problemas cada vez más altos" (Simondon, 2009, 317).

Es decir, para Simondon la vida se encaja como un dominio concreto del proceso de individuación.

A la hora de abordar la individuación vital, Simondon se hace eco del problema de medir el grado de "individualidad" de los seres vivos (cfr. Ibíd,230). Este problema evidencia que, manteniéndonos en un mismo dominio de individuación, podemos encontrar 
existencias más o menos individualizadas: existe, pues, una diferencia sutil entre "individuación" e "individualidad", referida, esta última, a la existencia más o menos separada de seres que, no obstante, pertenecen a una misma unidad de individuación ${ }^{3}$. Concretando más si cabe, la individuación vital se entiende como el marco donde se producen distintas individualizaciones. Estas individualizaciones se producen por procesos estrictamente vitales. Sin embargo, la aparición de lo psíquico planteará un escollo a esta individualidad puramente vital, a saber, el sub-individuo humano exigirá "herramientas" que no encuentra en lo vital, exigiendo el plano de lo "colectivo trans-individual" para efectuar su proceso de individualización.

Por otro lado, podemos observar cómo, al igual que Foucault, a la hora de abordar el problema de la individualización humana, Simondon parte de la vida desnuda. No obstante, en mi opinión, Simondon logra ofrecer una caracterización más satisfactoria de esta "nuda vida". El motivo lo encontramos en que, desde su explicación, se entiende cómo poder (biopolítico) y vida se encuentran necesariamente unidos en la constitución del sujeto humano, algo que Foucault entrevió pero que no exploró en profundidad (cfr. Agambem, 2007, 481-484).

Para Simondon, la vida está representada por la unidad vital del grupo, la cual ejerce un papel integrador y, al mismo tiempo, diferenciador respecto a los sub-individuos ${ }^{4}$ que la componen. El grupo es tomado como la "estructura" perteneciente al par "estructura-medio asociado", que surge como resultado de una individuación a partir de un sistema metaestable representado por el individuo físico. Así, las tareas de integración y diferenciación relativas al grupo respecto a sus sub-individuos, presuponen, a su vez, un medio asociado, entendido como la problematicidad del sistema de base (el individuo físico) (cfr. Simondon, 2009, 234)

En el individuo humano estas tareas de integración y diferenciación se corresponden, respectivamente, con la representación (como almacenamiento de los "gérmenes estructurales" del sistema de origen) y la actividad (como almacenamiento y amplificación de las energías potenciales, elementos desestructurantes relativos al medio asociado del sistema de origen). Así, la integración tiene que ver con el crecimiento de estructuración que se da en el proceso de individualización; y la diferenciación con el crecimiento de energías potenciales desestructurantes simultáneos a esa integración. La transducción final, esto es, la constitución de un individuo como sub-individuo de un grupo, su creciente individualidad, se produce por la afectividad de este sub-individuo que regula los elementos anteriores de diferenciación e integración. Es así como se entiende el proceso de individualización estrictamente vital en el ser humano.

3 Es necesario, por tanto, hacer hincapié en que, para Simondon, la individuación psico-colectiva, no es, estrictamente una individuación que tenga como resultado la constitución de un ser individuado, sino la creciente individualización que se produce dentro de la individuación vital.

4 Simondon en ningún momento utiliza la noción sub-individuo para referirse a los componente integrantes de la unidad vital representada en el grupo, en su lugar utiliza "individuo" para referirse tanto al grupo como a sus distintos constituyentes. En pos de la claridad expositiva, me permito la licencia de hablar de "sub-individuos" para referir a los componentes del grupo vital e "individuo" para referir a la unidad vital en tanto que tal, la cual toma Simondon como "individuo real". 
Para Simondon, el mixto entre representación integradora y acción diferenciante se produce en la reactividad de la acción del sub-individuo vivo por la cual éste aprecia su propia acción a través de la afectividad:

En este sentido existiría una profunda trialidad del ser viviente por la cual se hallarían en él dos actividades complementarias y una tercera que realiza la integración de las precedentes, al mismo tiempo que su diferenciación mediante la actividad de recurrencia causal; la recurrencia no añade, en efecto, una tercera función a las precedentes, pero la cualificación que autoriza y constituye aporta una relación entre actividades que no podrían tener ninguna otra comunidad. La base de la unidad y de la identidad afectiva está por tanto en la polaridad afectiva gracias a la cual puede haber allí relación entre (...) la diferenciación y la integración (Ibíd., 237).

La acción diferenciante y la representación integradora se integrarían, a su vez, en la recurrencia causal de la afectividad por la cual el sub-individuo aprecia su propia acción.

Dada esta función fundamental de la afectividad, Simondon señala que, gracias a ella, el ser viviente mantiene su unidad, eso sí, una unidad transductiva siempre amplificante (cfr. Ibíd., 239). Este mixto entre elementos diferenciantes y elementos integradores da cuenta de la "doble naturaleza" del sub-individuo (cfr. Ibíd., 252). Esta doble naturaleza se manifiesta en una dualidad funcional propia del sub-individuo, el cual es tomado como un elemento desestructurante del grupo o individuo en sentido estricto pero, a su vez, como un elemento que realiza, a través de esa acción diferenciante, la transducción del grupo a una unidad distinta que a la vez se diferencia y continua la unidad de base.

La unidad vital es, pues, concebida por Simondon como una estructura dada a una problemática -de origen físico- que tal estructura resuelve y a la vez amplifica con el crecimiento de individualidad de los sub-individuos que componen tal unidad vital. Este concepto de vida como una unidad transductiva en problematicidad creciente es el que en este artículo tomaremos como base para entender lo biopolítico como un poder ejercido sobre la vida. No obstante, para comprender como opera la estructuración biopolítica sobre la vida, hemos de detenernos en cómo el proceso de individualización implícito en la unidad vital puede prolongarse hasta desbordar tal unidad y exigir una nueva dimensión de individuación.

\subsection{La individuación psico-colectiva ${ }^{5}$}

En referencia al proceso de individuación psico-colectiva, Simondon utiliza el mismo esquema que usa para describir el proceso total de individuación. Roberto Espósito lo explica del siguiente modo:

Del mismo modo en que la individuación biológica del organismo viviente constituye la prolongación, en otro nivel, de la individuación física inacabada, a su vez, en un

5 Como ya hemos señalado en otras ocasiones, hay que tener presente que, para Simondon, la individuación psíquica y colectiva no son propiamente una individuación sino una “individualización” que efectúa, amplificando, la individuación vital, es decir, como un proceso de individualización que desborda la unidad puramente vital. 
nivel más alto, la individuación psíquica, específicamente humana, se inscribe, a distinta altura, en el punto de indeterminación de la individuación biológica que la precede (Esposito, 2006, 288-289).

La unidad vital viene garantizada por la complementariedad entre la acción diferenciante y el conocimiento integrador, propios ambos, de los sub-individuos que componen esa unidad. El origen de lo psíquico Simondon lo encuentra, precisamente, en el momento en el que el ser individuado vivo es incapaz de resolver la problemática que le plantea esa dualidad establecida entre la acción diferenciante y la percepción integradora, esto es, en el momento en el que su proceso de individualización alcanza un punto tal que supera el marco vital y exige un nuevo plano de individuación para efectuarse:

Hay psiquismo cuando lo viviente no se concretiza completamente y conserva una dualidad interna, [...] pero cuando la vida, en lugar de poder abarcar y resolver en unidad la dualidad de la percepción y de la acción, deviene paralela a un conjunto compuesto por la percepción y la acción, lo viviente se problematiza (Simondon, 2009, 241).

Será desde este papel que juega lo psíquico en el proceso de individuación desde el que comprender la relación entre poder y vida no lograda en la propuesta de Foucault. Desde la descripción de lo vital que Simondon ofrece, junto a esta noción de lo psíquico como la puesta en primera plana de la problematicidad inmanente a lo vital, podemos entender cómo es el poder -entendido como estructuración de la vida desnuda- lo que precisamente efectúa la individuación vital a raíz de la individualización del ser vivo humano que la problematiza exigiendo un nuevo plano de individuación -el colectivo-transindividual- el cual, no obstante, no tiene otra función que la de recuperar la unidad perdida a nivel vital 6 .

Como hemos visto, la dualidad percepción-acción se resuelve, en el dominio de lo vital, en una unidad transductiva a través de la acción relacional acometida por la afectividad. Es cuando esa dualidad de base no se resuelve en la unidad afectiva, cuando se produce el recurso al psiquismo. En ese momento la afectividad deja de ocupar un lugar central en lo viviente para organizarse junto con funciones perceptivo-activas, inaugurando una nueva problemática entre afectividad y emoción (cfr. Ibíd.,). Esta es la diferencia esencial entre la pura vida, y la vida psíquica: en la primera, la afectividad juega un papel central de dotación de unidad transductivo-resolutiva; en la segunda, la afectividad "se desdobla" generando problematicidad en lugar de resolución.

Así, para Simondon, el recurso al psiquismo es una "ralentización" de la individuación vital, un elemento de desestructuración de la individuación alcanzada a nivel de lo vital: "El psiquismo aparece o al menos es postulado cuando el ser viviente ya no posee en sí mismo el suficiente ser como para resolver los problemas que le son planteados" (Ibíd., 243). El psiquismo introduce una problemática "más alta", irresoluble al nivel de lo vivo. Se trata, como dice Simondon, de "una nueva inmersión de la individuación vital en la realidad preindividual” (Ibíd., 242).

6 Por otro lado, como este poder, en tanto que motivado por lo psíquico, se genera a partir de la propia vida, no aparece como un elemento externo a la misma sino que se trata de una exigencia inmanente a ella. 
Lo psíquico invalida la unidad alcanzada a nivel de lo vital. Al romper esta unidad, la individuación ha de alcanzar tal unidad a un nivel superior: el colectivo-transindividual. En tanto que lo psíquico es el encargado de poner en primera plana la problemática que la unidad vital lastraba y que recibirá su resolución a nivel de lo trans-individual, Simondon denomina a lo psíquico con el título de "transindividual naciente". Así,

El ingreso en la realidad psíquica es un ingreso en una vía transitoria, pues la resolución de la problemática psíquica intraindividual (la de la percepción y la de la afectividad) conduce al nivel de lo transindividual; las estructuras y las funciones completas que resultan de la individuación de la realidad preindividual asociada al individuo viviente sólo se cumplen y se estabilizan en lo colectivo (Ibíd., 244).

Este colectivo es la realidad transindividual, la cual Simondon no tarda en disociar de lo "social puro" y de lo "interindividual puro" pues, lo social y lo interindividual puros se identifican con la unidad vital representada por el grupo. De este modo lo social y lo interindividual existen de entrada en la individuación vital. Será precisamente la ruptura de esa sociedad como unidad vital por parte de lo psíquico la que exija una unidad ulterior al nivel de lo colectivo transindividual.

Lo colectivo trans-individual es definido por Simondon como el conjunto de referencia de las distintas individualizaciones en el seno de una unidad vital rota por la entrada de lo psíquico: se trata de una nueva unidad que repara la vital rota. Como Simondon señala: "Lo colectivo es aquello que resulta de una individuación secundaria respecto a la individuación vital, que retoma lo que la primera individuación había dejado de naturaleza bruta no empleada en lo viviente" (Ibíd. 464). Efectivamente, la individuación vital supone cierta estructuración de la naturaleza pre-individual física como ya hemos visto. Sin embargo, con la introducción de lo psíquico, esa "naturaleza bruta" que es estructurada en la individuación vital y que se encuentra latente en esta, se pone en primera plana, exigiendo una estructuración más allá de lo vital en lo colectivo-transindividual.

Lo colectivo-transindividual radica, pues, en la unidad que modela la individualidad de los seres humanos, la cual no podría alcanzar efectuación sin ello. Tal y como Esposito señala: "para que la vida psíquica pueda actualizar su propio potencial preindividual, debe impulsarlo al nivel de lo transindividual, es decir, debe traducirlo y multiplicarlo en la sociabilidad de la vida colectiva" (Esposito, 2006, 291). Lo psíquico pone en primera plana una problematicidad inmanente a la individuación vital, que esta no puede resolver desde la unidad vital alcanzada, sino que exige la aparición de lo transindividual como estructura resolutiva a tal problemática.

Regresando a las consideraciones de Foucault acerca del poder, el francés concebía la biopolítica como un control ejercido por el poder sobre la propia vida de los individuos, estructurando la misma de acuerdo a dispositivos. Tras este repaso por el proceso de individuación vital, observamos como la estructuración del suelo problemático en el que se convierte la unidad vital tras la aparición de lo psíquico viene garantizada por la referencia a lo colectivo trans-individual hacia el que se dirige la acción diferenciante del sub-individuo. Podemos por tanto decir que la biopolítica, entendida como estructuración o control de la vida es un aspecto inseparable de la vida en tanto que esta, para alcanzar su unidad perdida por la aparición de lo psíquico, refiere las distintas individualizaciones que 
se dan en su seno a una unidad que va más allá de lo puramente vital, a saber, lo colectivo: la vida, para resolver su problemática interna, necesita la referencia a la estructura colectiva que ejerce un poder estructurante -biopolítico- sobre sí misma.

Así observamos cómo, desde la propuesta de Simondon referente al proceso de individuación, podemos hacer explícita la relación que el poder entabla con la vida en el proceso de subjetivación y que le confiere a aquel, tal y como Foucault lo concibe, su naturaleza biopolítica: el poder biopolítico aparece como una estructuración o modelación de la vida, que se da en base al dominio de lo colectivo ${ }^{7}$. Ahora bien, la preocupación que espolea a Foucault en su análisis del poder es una preocupación por los procesos de subjetivación, no por los procesos de individuación. Sin embargo, como hemos señalado, la individuación psico-colectiva, estrictamente, no se trata de un proceso de individuación, sino de un proceso por el cual dentro de una individuación ya efectuada -la vida- pueden producirse procesos de individualización que amplifican esa individuación ya alcanzada. En mi opinión, esos procesos de individualización se corresponden con los procesos de subjetivación que Foucault adscribe a los mecanismos del poder y en ese sentido también parece entenderlo Combes: "pour autant, Foucault ne parle pas d'une objectivation des sujets, au sens d'une réification seconde d'êtres existant toujours-déjà comme sujets, mais de modes d'objectivation "qui transforment les êtres humains en sujet" (Combes, 2011, p. 56) Los procesos de subjetivación se entenderían como una cualificación de la vida desnuda. Esta cualificación, desde Simondon, se entendería como el recurso al plano de lo colectivo trans-individual para efectuar la individualización del ser humano.

De este modo, a partir de las reflexiones de Simondon, alcanzamos una definición del proceso de subjetivación como el proceso de individualización del sub-individuo vivo que exige el recurso a lo colectivo, al tiempo que comprendemos la relación que entablan en ese proceso la vida y el poder entendido, este último, como ese recurso a lo colectivo que permite la efectuación del proceso de individualización. Por último, dado que el proceso de individualización es intrínseco a la propia vida y dado que es en base a él -a su efectuación- por lo que se produce el recurso a lo colectivo en el cual, junto a la operación estructurante que este colectivo ejerce sobre lo vital desnudo, ciframos aquí el poder, observamos como poder y vida constituyen una díada inseparable en el proceso de subjetivación.

No obstante, en Foucault encontramos un enriquecimiento del modo en el que se producen las distintas individualizaciones cuya dinámica hemos explicado desde Simondon. Para Simondon, con la introducción de lo psíquico, la unidad vital se rompe y, para recuperar tal unidad es necesario el recurso a lo colectivo-transindividual. Sin embargo, los apuntes de Simondon respecto a la caracterización de lo "trans-individual" sólo inciden, en mi opinión, en su pertenencia a un nivel superior de individuación respecto a la vital y a su carácter de conjunto de referencia de las distintas individualizaciones vitales que, con la introducción de lo psíquico no pueden realizarse desde la afectividad. Sin embargo, de la mano de Foucault, podemos discernir que lo colectivo-transindividual viene representado por la red de dispositivos en los cuales el francés cifra todo proceso de subjetivación.

7 Un ejemplo de lo colectivo y de su papel estructurante lo podemos encontrar en el análisis que Simondon realiza sobre el mito y la creencia, cfr. Simondon, 2009, 445-446 
Para Agamben los dispositivos de los que nos habla Foucault, implican, siempre, un proceso de subjetivación, deben producir su sujeto. El término dispositivo refiere a un conjunto heterogéneo cuyo fin es el gobierno de la individualidad de los hombres (cfr. Agamben, 2011, 257). En definitiva, los dispositivos suponen un control de la individualización de los distintos individuos, por lo que, en Foucault encontraríamos un ahondamiento en ese proceso de individualización que Simondon describe: lo colectivo operaría su poder estructurante sobre la vida a través de los dispositivos y en este control de los dispositivos sobre lo vital se manifestaría el carácter biopolítico del poder.

Dado que para Simondon toda individuación psico-colectiva apela a lo colectivo transindividual y dado que tal colectivo, como presume este artículo, ha de entenderse como el efecto de los dispositivos sobre la nuda vida, no es posible entender una forma de individuación colectiva al margen de los mismos, al menos, desde un planteamiento estrictamente simondoniano y foucaultiano.

Así pues, desde la noción de vida simondoniana que aquí hemos tratado de ofrecer, encontramos una vía para poner en relación lo que Deleuze consideraba el tercer eje del proceso de subjetivación, esto es, la vida, con el eje del poder. Para poder dar una forma completa a este proyecto, no obstante, sería necesario descubrir el papel que toma el saber en este proceso de subjetivación y su relación tanto con el eje de la vida como con el eje del poder. En mi opinión la segunda tarea, esto es, la relación saber-poder es la aportación fundamental del pensamiento de Foucault. Por su parte, la relación entre saber y vida queda magistralmente expuesta en el ya citado escrito de Agamben "La inmanencia absoluta".

A continuación nos detendremos, sin embargo, en la consideración de Foucault acerca del posible carácter negativo de la estructuración biopolítica. En efecto, para Foucaul, el poder biopolítico implica la renuncia a toda forma de creatividad en la medida en que supone un control exhaustivo de la individualidad humana que la condena a unos límites muy estrictos, fuera de los cuales no es posible entender individualidad alguna. A mi modo de ver, efectivamente la estructuración biopolítica implica tales elementos negativos. Sin embargo, como trataremos que demostrar en lo que resta, tales elementos negativos no son intrínsecos a la estructuración biopolítica en tanto que tal, sino que por el contrario, evidencian que tal estructuración puede presentar un carácter patológico derivado.

En los apartados siguientes, pues, debemos detenernos ante la posibilidad de una biopolítica patológica, partiendo, eso sí, de la inevitabilidad de lo biopolítico y, por ello, del carácter derivado de sus elementos patológicos, todo ello, desde la noción simondoniana de vida ya alcanzada y de la comprensión de la relación entre poder y vida que hemos tratado de establecer.

\section{El carácter patológico de la biopolítica}

Como hemos señalado, la individuación psico-colectiva implica tanto la estructuración de un suelo pre-individual -la vida- como el mantenimiento de la problemática presente en ese suelo y que la estructuración debe mantener como motor para nuevas individuaciones. La individuación colectivo-transindividual, por tanto, ha de ser considerada como la restitución de un equilibrio meta-estable perdido a nivel de lo vital tras la irrupción de lo psíquico. La individuación colectiva es vista, pues, como una estructura y el medio asociado a tal estructura que se encuentra representado por la problematicidad de su sistema individuante de origen: la vida. 
Desde este planteamiento, la estructuración biopolítica llevada a cabo en base a lo colectivo-transindividual corre dos riesgos: o bien que su aspecto desestructurado relativo a su medio asociado impida la consumación de toda estructura; o bien, que sus elementos estructurales acaben inmunizándose respecto a su medio asociado. Todo sistema biopolítico entendido como estructuración de un suelo vital problemático implica (cfr. Simondon, 2009, 492-501) elementos estructurales que le dan consistencia como sistema. A estos elementos Simondon los denomina "normas". Sin embargo el proceso de individuación del cual tal estructuración es resultado, implica un "valor" que dichas normas deben respetar para no anularlo y que implica que tales normas alberguen en su seno su "propia destrucción" o transformación en nuevas normas estructurales.

Para Henri Bergson (cfr. Bergson, 1962, 72-95), muy afín a Simondon en este punto, el conjunto de "normas" que componen el sistema colectivo ejercen una presión sobre los distintos individuos que componen una sociedad. Se trataría de lo que el francés considera la fuente de la "moral cerrada". Las normas de tal sistema actuarían en el individuo a modo de "instintos" de conservación del grupo. Frente a esta moral cerrada, existe otro tipo de moral que encuentra su fuente en una suerte de estado emocional o "amor a la humanidad". Esta otra moral no se circunscribe ni depende de normas algunas, al contrario, se trata de una "moral abierta" que impulsa a los individuos a una aspiración moral sin objeto determinado, sino la humanidad en su conjunto. Esta moral abierta puede, para Bergson cristalizar en normas concretas que constituyan una moral cerrada, pero no se agota en ellas. Moral abierta y moral cerrada no se excluyen en el planteamiento de Bergson, sino que ambas se encuentran relacionadas, del mismo modo que el valor y las normas lo están en el planteamiento de Simondon.

Para Simondon las normas que componen un sistema colectivo determinado son la solución provisoria a una problemática vital radical. La emoción de la que Bergson hace depender la moral abierta, en mi opinión, puede corresponderse con la idea de "valor" manejada por Simondon, y que se define como esa creciente amplificación de la problemática radical que conduce a nuevas formas de individuación y que las normas deben respetar ${ }^{8}$.

Simondon cree encontrar, pues, un elemento normativo inmanente al propio proceso de individuación que, a nosotros, nos permite diferenciar entre una forma "positiva" de biopolítica y una forma "negativa" o patológica. Para el francés, una ética adecuada al proceso de individuación implica el respeto del juego que se establece entre normas y valor: toda evaluación normativa acerca de las normas constitutivas de un sistema cualquiera ha de fundarse en si tales normas son contrarias o no al proceso de individuación, esto es, si lo anulan o lo respetan. Roberto Espósito en su obra Bios se hace eco de este criterio norma-

8 Pese a la coincidencia entre el planteamiento bergsoniano y el simondoniano, existe una diferencia fundamental entre ellos. Tal diferencia la encontramos en la consideración de la naturaleza. En mi opinión Bergson maneja una concepción de la naturaleza que lo lleva a considerar la moral abierta como resultado del "genio humano" que rompe con el impulso natural a la conservación de una determinada sociedad; mientras que Simondon concibe a la naturaleza como physis, esto es, como presentando dos dimensiones una extensiva y otra intensiva. Las normas que constituyen un sistema colectivo se deberían a esa dimensión extensiva de lo natural, mientras que el "valor" presente en el proceso de individuación y constitutivo (en términos bergsonianos) de una moral abierta, tendrían que ver con el envés intensivo de la naturaleza. De este modo, la moral abierta no depende de "genio humano" alguno, si de la naturaleza como physis. 
tivo. Para el italiano, según el proceso de individuación descrito por Simondon, "el hombre nunca pierde su vínculo con su ser viviente" (cfr. Esposito, 2006, 286), siendo en base a esta conexión por lo que podremos establecer un criterio normativo que permita distinguir entre formas patológicas y positivas de biopolítica.

La forma patológica de biopolítica se entiende como una "inmunización" de la estructura colectivo-transindividual y de la estructuración biopolítica que ejerce sobre el suelo vital. En otras palabras, la biopolítica se convierte en patológica cuando la estructura colectiva en base a la cual se ejerce el control biopolítico, se blinda respecto al medio asociado a ella, el cual debería motivar su constante transformación. A través de este blindaje, la estructura colectiva pretende "mantenerse viva". Sin embargo, como señala Esposito, "para la vida, la única manera de aplazar la muerte no es conservarse como tal, quizás en la forma inmunitaria de la protección negativa, sino renacer constantemente de maneras diversas" (Esposito, 2006, 291). Es decir, un sistema de normas - esto es, lo colectivo en base al que se efectúa la estructuración biopolítica de un suelo vital problemático- que se inmunice respecto a la problemática vital, está auspiciando su propia muerte, se sitúa como contrario a la vida, pues ésta se entiende como la sucesión de nuevas formas de estructuración y, por tanto, aparece como una estructuración patológica.

Así pues, el juego que se establece entre normas y valor en Simondon, para Esposito, supone el mantenimiento del sistema de normas en la apertura radical que le es propia:

El único valor que permanece estable, en la transición de las normas desde un sistema hacia otro, es la conciencia de su traducibilidad en formas siempre distintas y necesariamente transitorias. El modelo normativo más acabado es, incluso, el que ya prefigura el movimiento de su propia deconstrucción en favor de otro posterior. (Ibíd., 301)

Desde este criterio normativo que se desprende del proceso de individuación de lo real, podemos evaluar si una determinada estructuración biopolítica es o no patológica: si tal estructuración impide cualquier atisbo de transformación o novedad, nos encontramos ante una biopolítica patológica. A continuación nos detenemos en este aspecto patológico de la biopolítica.

\section{Biopolítica y biopoder: la inmunización como proceso patológico de la estructura biopolítica}

Cuando Foucault habla del Estado de Policía como la característica principal de la práctica gubernamental basada en la Razón de Estado (cfr. Foucault, 2009, 18-25), nos encontramos con la descripción de una estructuración biopolítica patológica que busca la inmunización respecto a su "medio asociado" representado por la vida problemática.

Esposito, en su obra Comunidad, inmunidad y biopolítica, realiza una rica explicación acerca del proceso de inmunización por el cual un sistema colectivo se blinda respecto al suelo "común" que supone el medio asociado de toda estructura colectiva trans-individual y que permite tanto la constante individuación de tal estructura como la individualización de los individuos que entraña. El proceso de inmunización del sistema se refleja en una libe- 
ración por parte del individuo respecto de su "contingencia ambiental" -"medio asociado" a la estructura psico-colectiva según nuestra terminología-. Así, a través del proceso de inmunización, "el individuo es inducido a cerrar su originaria apertura y a circunscribirse a la esfera de su interior" (Esposito, 2009, 85). Es decir, un sistema inmunitario se cierra sobre su carácter de estructura, anulando el suelo pre-individual y problemático sobre el que se yergue. En tanto que ese suelo es el motor para nuevas transformaciones en el sistema, éste, a través del proceso inmunitario, se absolutiza.

Foucault señala que, con la entrada en la Época Clásica, el antiguo derecho de vida y muerte que regía la sociedad, y que se manifestaba en el derecho del soberano de "hacer morir o dejar vivir", pierde vigencia, operando el poder, no ya a través de la supresión de la vida sino de su potenciamiento (cfr. Foucault, 2005, 165-7). El objetivo biopolítico, esto es, la estructuración de la vida, se lleva a cabo a través de un procedimiento que sigue siendo igualmente patológico que aquel basado en el derecho de vida y muerte. El viejo poder de hacer morir cede ante un poder que administra los cuerpos y gestiona la vida, buscando "la sujeción de los cuerpos y el control de las poblaciones" (Ibíd., 169). Foucault denomina a este nuevo poder "biopoder". Así, la estructuración biopolítica operaría patológicamente, esto es, de manera inmunitaria, a través de un "biopoder" que potencia la vida para su posterior control.

Es en este proceso donde Esposito encuentra el carácter inmunitario del sistema colectivo patológico. Este biopoder operaría la "liberación del individuo" respecto de su suelo problemático a través de una progresiva inclusión de ese suelo en el propio sistema:

La relación entre sistema y ambiente [estructura colectiva y medio asociado] (...) no se resuelve a través de una simple reducción de complejidad ambiental, sino más bien mediante su transformación de complejidad externa a complejidad interna al sistema mismo. Pero a esta estrategia de interiorización activada por el proceso inmunitario se viene a unir una segunda aún más cargada de consecuencias disolventes de la diferencia ambiental, esto es, su integral inclusión en el interior del sistema o, dicho de otro modo, su objetiva eliminación. (Esposito, 2009, 85-86).

Toda individuación se correspondía con la actualización de un sistema individuante de base mediante la aparición de una singularidad. En el caso de la individuación colectiva, el sistema individuante es la vida misma, problematizada con la aparición de lo psíquico. La estructuración biopolítica patológica se entiende como una individuación colectiva a partir de un suelo vital pre-individual que, en lugar de mantener y amplificar ese suelo, se inmuniza respecto a él anulándolo. En la Época Clásica, mediante el biopoder, esta inmunidad se consigue, no anulando toda singularidad (como ocurría con el antiguo derecho de vida y muerte), sino favoreciéndola pero bajo las formas propias de tal sistema, por lo que, estrictamente, no permite que el acontecimiento (actualización de un suelo pre-individual) acontezca sino que, a través de una per-formación de tal acontecimiento, lo impide absolutamente.

Así pues la estructuración biopolítica, constitutiva de la subjetividad humana, puede tornarse patológica, cifrándose tales formas patológicas en una inmunización del sistema colectivo respecto a su suelo problemático. La búsqueda de una biopolítica positiva que autores 
como Hard y Negri (Hard y Negri, 2009), o el propio Esposito (Esposito, 2006), han perseguido ha de dirigirse, precisamente a ese carácter abierto que toda estructura biopolítica ha de presentar, si bien, sin obviar el elemento estructural que toda biopolítica presenta de hecho.

\section{Conclusión}

A través de una explicitación del proceso de individuación de lo real, Simondon nos otorga una nueva noción de "vida" desde la cual, la estructuración biopolítica entendida como la individuación psico-colectiva se observa como un elemento necesario en la constitución de la subjetividad humana. A su vez, este proceso de individuación de lo real puede proporcionar un criterio normativo desde el cual comprender cómo una estructuración biopolítica puede tornarse patológica a través de un proceso de inmunización respecto a su suelo problemático.

En mi opinión esta noción simondoniana de vida puede resultar de suma relevancia para acometer el proyecto señalado por Deleuze y acometido por Agamben: introducir a la vida como tercer eje en la constitución de la subjetividad humana, junto a los ya estudiados por Foucault del saber y el poder. En concreto, dada la naturaleza biopolítica del poder, tal y como Foucault lo concibe, se hace necesario un análisis de lo puramente vital que permita comprender su asunción por parte del poder y, al mismo tiempo, la relación de ambos elementos en los procesos de subjetivación. En este sentido, Simondon otorga una perspectiva inédita en el planteamiento estrictamente foucaultiano: una concepción de la vida como dominio de individualizaciones motivadas por su propio carácter problemático o metaestable. Desde esta concepción, el poder, entendido como estructuración o control de la nuda vida -definición que asume el propio Foucault- no aparece como un elemento externo a la vida, sino como inmanente a la misma y necesario para su correcto desenvolvimiento.

La noción de individualización, como un crecimiento progresivo de los sub-individuos que componen la unidad vital es equiparable a lo que Foucault denomina "procesos de subjetivación", si bien es necesario introducir una importante diferencia. Para Simondon, el proceso de individualización puramente vital como el proceso de individualización humano reciben un tratamiento semejante con la única, aunque importante diferencia de que el segundo excede el dominio de lo puramente vital apelando, para su realización, al dominio de lo colectivo trans-individual.

A mi modo de ver, este segundo proceso de individualización que ya no puede realizarse en el terreno de la "nuda vida" es el que se corresponde estrictamente con el proceso de subjetivación analizado por Foucault. En este sentido, Foucault habría introducido una noción fundamental al planteamiento de Simondon referida a cómo opera lo colectivo trans-individual sobre la nuda vida: la noción de dispositivo. Foucault habría analizado el modo de operar de este poder biopolítico sobre la vida pero careciendo de una noción de vida tal que amparase ese operar. Desde la propuesta de Simondon esa carencia se suple a través del establecimiento de una noción de vida desde la cual comprender mejor la naturaleza biopolítica del poder.

Este proyecto foucaultiano acerca de los procesos de subjetivación requiere, para cobrar un carácter completo, que la relación aquí establecida entre vida y poder, se complemente con la relación que ambos ejes mantienen con el saber. Sin embargo, ese cometido excedería los objetivos de este artículo en el que sólo se ha querido contribuir a tal proyecto a través del establecimiento de una noción de vida coherente con el mismo. 


\section{Bibliografía}

Agamben, G. (2007): La potencia del pensamiento, Buenos Aires: Adriana Hidalgo.

Agamben, G. (2011): «¿Qué es un dispositivo?», Sociológica n 73, pp. 249-264.

Bergson, H. (1962): Las dos fuentes de la moral y la religión, Buenos Aires: Editorial Sudamericana.

Combes, M. (1999): Simondon : individu et collectivité. Pour une philosophie du transindividuel, París: PUF (Presses Universitaires de France)

Combes, M. (2011): La vie inséparée. Vie et sujet au temps de la biopolitique, París: Éditions Dittmar.

Esposito, R. (2006); Bíos. Biopolítica y filosofía, Buenos Aires: Amorrortu.

Esposito, R. (2009): Comunidad, inmunidad y biopolítica, Barcelona: Herder.

Foucault, M. (2005): Historia de la sexualidad (vol. I). La voluntad de saber, Madrid: Siglo XXI.

Foucault, M. (2009): Nacimiento de la biopolítica, Madrid: Akal.

Hard, M. y Negri, A.(2009): Commonwealth. El proyecto de una revolución del común, Madrid: Akal.

Simondon, G. (2009): La individuación a la luz de las nociones de forma e información, Buenos Aires: Cactus. 